\title{
Zur Überlieferung von ahd. riuti und riutî
}

Prinz, Michael

DOI: https://doi.org/10.1515/9783110526585-013

Posted at the Zurich Open Repository and Archive, University of Zurich ZORA URL: https://doi.org/10.5167/uzh-160729

Book Section

Published Version

Originally published at:

Prinz, Michael (2018). Zur Überlieferung von ahd. riuti und riutî. In: Czajkowski, Luise; Ulbrich-Bösch, Sabrina; Waldvogel, Christina. Sprachwandel im Deutschen. Berlin/Boston: De Gruyter, 159-167. DOI: https://doi.org/10.1515/9783110526585-013 


\section{Michael Prinz \\ Zur Überlieferung von ahd. riuti und riutī}

An der erstaunlichen Vielzahl plesionymer Rodungsbezeichnungen, die in den germanischen Sprachen in Gebrauch waren (vgl. dazu Prinz i. Dr.), hat die Überlieferung des Althochdeutschen einen erheblichen Anteil. Bei nicht wenigen dieser Bildungen sind jedoch das Alter und die Überlieferungszusammenhänge noch einigermaßen unklar. So ist beispielsweise ein Appellativum «riuti〉 nach Ausweis der Wörterbücher (vgl. Starck/Wells, Gl.-Wb. 1, 489; Schützeichel, Gl.-Wortsch. 7, 449) für das Spätalthochdeutsche des 11. Jahrhunderts dreimal bezeugt, wobei bei näherer Betrachtung alle Nennungen mit dem sog. Mondseer Bibelglossar (M) (dazu ausführlich Meineke 2009; 2013) und der Benediktinerabtei Tegernsee zusammenhängen.

Der älteste Beleg findet sich im Clm 19440, einem frühen Textzeugen der Bibelglossatur. Die Schriftheimat der in der Handschrift um 1000 eingetragenen Kontextglossen ist unklar, der Codex dürfte jedoch bald nach der Jahrtausendwende nach Tegernsee gelangt sein (sofern er nicht ohnehin hier entstand) (zur Hs. vgl. Davids 2000, 45; BStK Nr. 665). Unter den Glossen zu Jeremias findet sich darin ein Eintrag Nouale nivt; interlinear wurde von einer anderen als der Texthand dazu ród. t rivti ergänzt. ${ }^{1}$ Aus dieser Handschrift schöpfte später, wohl im dritten Jahrhundertviertel, der in Tegernsee entstandene Clm 18140. Hier taucht riuti erneut auf - nunmehr als Kontextglosse (ród bleibt interlinear). ${ }^{2}$ Aus dem Clm 18140 wurde schließlich am Ende des 11. oder Anfang des 12. Jahrhunderts eine weitere Handschrift abgeschrieben (vgl. BStK Nr. 1623; Wunderle 2010, 203), von der jedoch nur drei Doppelblätter erhalten sind. Eines davon überliefert die Glossen zu Jeremias, darunter auch wieder: Nouale. niv lenti. $t$ rivti. ${ }^{3}$ Alle drei Belege geben damit letztlich denselben volkssprachigen ${ }^{4}$ Akk.Sg. aus Jer. 4,3 (novate vobis novale) wider. ${ }^{5}$

1 Pag. 348; Digitalisat: urn:nbn:de:bvb:12-bsb00036881-8 (Bildnr. 356). Edition: StSGl. 1, 627, 4 und Davids (2000, Nr. 994).

2 Fol. 192r (neue Zählung); zur Hs. vgl. BStK (Nr. 637). Digitalisat: urn:nbn:de:bvb:12-bsb000065258 (Bildnr. 389); Edition: StSGl. 1, 627, 3. Zudem wurde ród als $f$ (rancice) markiert, dazu Schröder $(1957,195)$.

3 Cgm 5248,2 (3. Doppelblatt), fol. 1vb [übergeschrieben: $f$ (rancice) rod.]; zur Hs. vgl. BStK (Nr. 846) und Wunderle (2010, 203, 205-206). Edition: Hartl (1930, 96), dort irrtümlich: „fol. $2^{\text {vb“. }}$

4 Die Tegernseer Textzeugen der Mondseer Bibelglossen zeigen zwar bairischen Sprachstand, insgesamt ist aber „von verwickelteren Verhältnissen einer mehrschichtigen oberdeutschen Überlieferung“ auszugehen (Meineke 2009, 629).

5 Zur Interpretation der Stelle vgl. Tiefenbach (1980, 303): „die Zuschauer auffordern, ihr Inneres zu säubern, wie ein Stück Land zur Aussaat bereit gemacht wird, indem man Unkraut und Gestrüpp entfernt“. 
Bereits deutlich früher erscheint 〈riut(i)〉 als Kompositionsglied in Zusammensetzungen wie riutachus ${ }^{6}$ 'sarculum', rivtisegans $a^{7}$ 'falcastrum' und niuriuti 'novales'8. In den Editionen und Wörterbüchern ist das betreffende Morphem meist kommentarlos einem Lexem ahd. riuti st. N. (ja) 'urbar gemachtes Land, Rodung' zugeordnet (neben Starck/Wells, Gl.-Wb. und Schützeichel, Gl.-Wortsch. vgl. etwa Schatz 1907, §16a). Ein solches ja-stämmiges Rodungswort ist im Althochdeutschen auch tatsächlich nachweisbar, v. a. toponymisch. Geminationsgraphien und -e im Dat.Sg. bei Belegen wie den folgenden stützen jedenfalls ein entsprechendes Lemma:

778 (Kopie 2. Viertel 9. Jh.) DE RIUTTE, az Riutte ${ }^{9} ; 830$ in loco quae dicitur ad Riute ${ }^{10}$; um 976 ad Rîute $^{11}$. Belege auf $-i$ werden meist entsprechend als nominativische Nennformen des ja-Stamms aufgefasst (so etwa bei Schatz 1907, §96e und Wiesinger 1992, 366): z. B. 788-790 (zu 748-757; Kopie Mitte 12. Jh.) in pago Filusgaoe in loco qui vocatur Riuti [Var. 13. Jh. Ruite] ${ }^{12}$; 857-864 in loco qui dicitur Riuti ${ }^{13}$; um 925 (Kopie um 930) in loco Riutihouum nominato ${ }^{14}$; 10641065 (zu ca. 755-785; Kopie von 1521) census predii nomine Rǒti ${ }^{15}$.

Während das Neutrum dann im Urkundenkorpus des 13. Jahrhunderts nachweislich im West- wie im Ostoberdeutschen begegnet, ${ }^{16}$ sind viele frühe «riuti〉-Belege, auch

6 StSGl. 1, 619, 48: Isaias; Rb (alem., Ende 8./Anfang 9. Jh.); vgl. BStK (Nr. 296).

7 StSGl. 2, 253, 19: Gregor; Wien, Cod. 2723 (bair., 2. Hälfte 10. Jh.). Die „Normalform“ riuti- mit Fugenvokal 〈i (dazu Gröger 1910, 90, §52) ist indes spärlich bezeugt, wofür Gröger mögliche Gründe abwägt: Mischung mit dem neutralen $a$-Stamm mhd. riet (96, §56.3), lautliche Abschwächung zu 〈e〉 und analogische Tilgung (104-105, §60), Bezug auf eine verbale Basis (172, §115). Der älteste Beleg, St. Gallen, StiftsB, Cod. 299, pag. 261, stammt zwar noch aus der zweiten Hälfte des 9. Jhs. (vgl. BStK Nr. 225), lautet aber bereits riutsegansa. - Für eine Belegübersicht zu den betroffenen Bildungen (riutackus, riuthouwa, riutīsarn, riutsegansa) vgl. Schützeichel, Gl.-Wortsch. 7, 449-450 und Sehrt $(1962,160)$.

8 StSGl. 2, 411, 2: Prudentius; Rom, Vat. lat. 5821 (alem., 11. Jh.). Das bereits im 10. Jh. entstandene Bibelglossar des Codex St. Paul 82/1 übersetzt fol.188v (Buch der Sprüche) Innoualibus patrum mit alem. inniuriutin; zur Hs. vgl. BStK (Nr. 779). Edition: StSGl. 1, 526, 34. - Zu weiteren Belegen (alle alem., 11. und 13. Jh.) s. AWB 6, 1308. Von diesen bezeugt allerdings lediglich án demo níuríute in Np sicher ein st.N. Später hat auch Iwein 3285: an ein niuweriute (Hs. A niwe rute). In den Dialekten kommen allerdings beide Genera vor: vgl. Schweiz. Id. (6, 1804): „Neu-Rüt“ (N.), 1815: „,nicht selten [...] Neu-“ (F.). Zu mhd. niu-geriute vgl. Prinz (2010, 305 Anm. 93).

9 Trad. Freising Nr. 91 (= Niclasreuth, LK Ebersberg, Bay.); vgl. Puchner (1951, 64).

10 Trad. Freising Nr. 600 (= Ober-/Unterreith, LK Erding, Bay.); vgl. Baumann/Dachs (1989, 158).

11 ANBÖ 2, 865 (= Reith i. Alpbachtal, Tirol).

12 Lošek (1990, Nr. 5,2) (= Reith b. Reisbach, LK Dingolfing-Landau, Bay.).

13 Trad. Freising 805 (lt. Register wie Anm. 10).

14 Hauthaler/Martin (1910, 92) (= Reithofen, Gem. Pastetten, LK Erding, Bay.).

15 Trad. Wessobrunn Nr. 1; die „lautgeschichtlich“ begründete Gleichsetzung mit Rott (LK Landsberg, Bay.) bei Höppl $\left(1984,109^{\star}\right)$ ist haltlos.

16 Vgl. WMU 2, 1449: bair. daz reutt (Jeßnitz, Ndö.), alem. ze niderm rvtj (Freiburg i.Br.) etc. Vgl. auch Lexer (s.v. riute stn.); Schweiz. Id. (6, 1804): Rüt n. 'Rodung'. 
die appellativischen, ambig. Ob sie stets den ja-Stamm zeigen, ist durchaus unklar, da daneben - wie in zahlreichen vergleichbaren Fällen (vgl. Braune/Reiffenstein, Ahd. Gr. 2004, §201 Anm. 1) - ein feminines Abstraktum mhd. riute < ahd. riutī(n) existiert hat, welches von den Wörterbüchern ab dem 13. Jahrhundert weiträumig nachgewiesen wird. ${ }^{17}$ Entsprechend finden sich etwa in frühen bayrischen Urbaren Belege für daz Rævt (ca. 1250-1270) neben Feminina wie: von ainer nivwen rivt oder Vf der Rivte (1231-1234) (Klose 2003, 120; Heeg-Engelhart 1990, Nr. 1721b, 1726). Im alemannischen Sprachraum war $\operatorname{riutī}(n)$ hochfrequent und führte aufgrund der intensivierten Rodungstätigkeit (vgl. etwa TNB 1.1, 124-125) des Hochmittelalters zu einer unüberschaubaren Zahl von Orts- und Flurnamen. ${ }^{18}$

Etymologisch lässt sich riutī unschwer als Verbalabstraktum zu dem jan-Verb ahd. riuten 'roden, lichten' ${ }^{19}$ stellen, entsprechend dem Muster got. daupeins 'Taufe' zu daupjan 'taufen', ahd. restī 'Ruhe' zu resten 'ruhen' etc. Das Verb wiederum lässt sich angesichts der $e$-Stufe am ehesten mit Riecke (1996, 311-312) als denominale Bildung zu dem in riuti vorliegenden ja-Stamm auffassen. In mittelhochdeutscher Zeit konnte es dann durch den Zusammenfall von /iü/ und / $\overline{\mathrm{u}} /$ nach dem Muster von diuhen (< ahd. dūhen 'drücken') : dûhte zu einem analogischen Rückumlaut riuten : rûte kommen. ${ }^{20}$

Über das Alter des Femininums ist indes wenig bekannt. Dabei lassen sich deutlich frühere Belege beibringen, als es bislang nach Ausweis der Wörterbücher den Anschein hat. Eine prominente zeitgenössische Beschreibung des hochmittelalterlichen Landesausbaus findet sich in der frühmittelhochdeutschen Pflichtenlehre Vom Rechte, die von der Millstätter Handschrift, Klagenfurt, Landesarchiv, Geschichtsverein f. Kärnten, Sammelhs. 6/19, fol. 135v-141v, unikal überliefert wird. Der vermutlich um die Mitte des 12. Jahrhunderts entstandene Text - die Handschrift wurde am Ende des 12. Jahrhunderts auf südbairischem Gebiet angefertigt (vgl. Ganz 1989, 1054; Hamano 2016, XX) - beschreibt anschaulich in einem exemplum für den tran-

$17 \mathrm{Vgl}$. bereits BMZ (s.v. riute): „auch ein riutî stswf. muss es gegeben haben“, daneben Lexer (s.v. riute stf.): 'Stück Land, das durch riuten urbar gemacht wurde', ${ }^{1} \mathrm{DWB}$ (s.v. Reute F.): 'ausgereutetes Stück Land', DRW (s.v. Reute): 'urbar gemachtes Stück Land, Rodung', Schweiz. Id. 6 (s.v. Rü̈ti f.): 'Rodung, von Holzwuchs, Buschwerk gereinigtes, urbar gemachtes Stück Land', WMU 2 (s.v. riute st.F.): ,Rodung, gerodetes Land', Schwäb. WB 5 (s.v. Reute I f.): 'Rodung, Neubruch', Schmeller 2 (s.v. Die Reut, Reuti): 'das Ausreuten'.

18 Die Datenbank auf www.ortsnamen.ch lieferte am 7.2.2017 für die Schweiz 1326 Treffer (Uf der Rüti, Untere Rüti etc.).

19 Nur in Gll. belegt (Schützeichel, Ahd. Wb. 2012, s.v.); vgl. aber Mitte des 11. Jhs. in einer in die Bamberger Alkuinbibel (StB Bamberg, Msc. bibl. 1) eingetragenen Traditionsnotiz: daz niugeuang unte daz holz daz man riutet (Prinz 2010, 304-306). Neben dem Präfixverb (Anfang 9. Jh., arriuton 'extirpauerunt' in Rf) steht das ebenfalls früh bezeugte Nomen agentis vrriutto sw. M. 'stirpator' in der Benediktinerregel (vgl. Seebold 2008, 684).

20 Lexer (s.v. riuten); vgl. dazu Paul, Mhd. Gr. 2007, §M89 Anm. 4 mit vergleichbaren „Rückumlauten“ (für liuhten, diuten, triuten, stiuren). 
sitorischen Charakter des irdischen Wohlstands ${ }^{21}$, wie meister und chneht gemeinsam ein Gebiet roden. Darin heißt es u. a.:

fol. 136v, Z. 11-13: fi enfamet hin gant. ein rǒtin beftant. fi rǒtent [m] it den armen; fol. 137r, Z. 25: da der meiftir unde der chneht. bedefamt hin gant. unde die rǒtin beftant. die chleinen ftoche. fi óz nement. unz fi an den grozzen choment; fol.137r, Z. 14-15: w [an lieze er] in da ftan. fo wære daz rǒtin ungetan; fol. 137r, Z. 24 - fol. 137v, Z. 2: da der meiftir unde der chneht. bede famt hin gant. unde die rǒtin beftant. fo ez danne zediv wirt. daz div rǒtin gebirt. ${ }^{22}$

Die Passage überliefert neben dem Verb rǒtent vier Belege für das Femininum rǒtin (1x Nom.Sg., 3x Akk.Sg.). Während die Wendung die rǒtin bestan noch die abstrakte Motivationsbedeutung erkennen lässt ('das Roden [als etw. Beschwerliches] auf sich nehmen'), wird im Text auch der substantivierte Infinitiv ${ }^{23}$ als Nomen actionis verwendet (daz rǒtin). Dieser scheint den Vorgang kontinuierlich, das Femininum eher punktuell zu konzeptualisieren (vgl. das Stoßen vs. der Stoß). Die semantische Kompatibilität mit dem Verb gebern indiziert dann bereits die Entwicklung einer konkreten Lesart.

Die Nichtberücksichtigung dieser frühen Belege in den Wörterbüchern mag mit ihrer etwas eigentümlichen Form zu tun haben, die sich jedoch leicht erklären lässt. Der Text verwendet konsequent 〈iv iù für mhd.-bair. /iu/ (hivte, gebivtet, uivr, div,

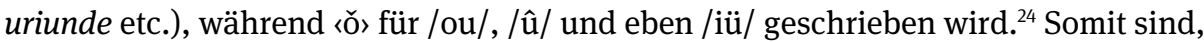
wie im östlichen Oberdeutschen $\mathrm{zu}$ erwarten ${ }^{25}$, der Diphthong/iu/ (gebivtet) und monophthongiertes [y:] < /iü/ (rǒtent) graphematisch klar geschieden. Dass Letzteres mit / $\hat{u} /$ (dessen Umlaut-Allophon mit /iü/ zusammengefallen war) und /ou/ gleichbehandelt wird, ist ein Reflex der im Südbairischen zu dieser Zeit stattfindenden Diphthongierung, welcher die Millstätter Handschrift mit den tentativen $\check{o}^{-}$ Schreibungen gerecht zu werden versucht. ${ }^{26}$

21 Zur Interpretation der kontroversen Passage vgl. Speicher $(1986,48-60)$ und Sullivan (2001, 108-116).

22 Zit. nach der handschriftennahen Ausgabe von Maurer (1965, 160, 162).

23 Sofern man nicht gerade daz zu diu korrigieren möchte (dazu Schröder 1891, 297), wofür es angesichts von behůtin im Text (ebenfalls jan-Verb mit Inf. -in) keine Notwendigkeit gibt.

24 Zur umlautbedingten Spaltung von spätahd. /iu/ - /iü/ vgl. etwa Kranzmayer (1956, §16) und Wiesinger (1970, 235-237). Die Textbelege folgen durchweg der dort genannten komplementären Verteilung (mit innerparadigmatischem Ausgleich bei den Klasse-II-Verben): z. B. ǒch, berǒbot, getǒbot, urǒwe, gelǒbet, tǒffe - ǒzzen, ǒz, schǒr, nachgebǒr, mǒrin, bǒwen - rǒtin, rǒtent.

25 Spätestens ab dem 13. Jahrhundert sind die Kontinuanten von spätahd. /iu/ und /iü/ meist mehr oder weniger deutlich geschieden; vgl. dazu Reiffenstein (2003, 2911-2912, Abschn. 5.3.2.2).

$26 \mathrm{Zu}$ bair. «ǒ̀ als einer ambivalenten Schreibung der frühen Diphthongierungsphase vgl. Reiffenstein (2003, 2910, 2912). Diese „fortschrittliche“ Bezeichnung der neuen Diphthonge ist - anders als Schneider $(1987,87)$ annimmt - in der Hs. ebenfalls noch recht mehrdeutig. Die Bemerkungen bei Speicher $(1986,50)$ zu einem angeblichen „Wechsel von iu und ou“ sind unbrauchbar. 
Morphologisch ungewöhnlich ist bei rǒtin allerdings der Ausgang auf «in〉 im Nom./Akk.Sg. Die femininen Verbalabstrakta auf -ì(n) stellen ursprünglich starke -ini-Bildungen dar (zu jan-Verben), die entsprechend noch im Gotischen als i-Stämme flektiert werden (z. B. daupeins, ahd. toufi) (vgl. etwa Krahe/Meid 1969, 117-118). Im Deutschen sind sie früh mit den schwachen Adjektivabstrakta des Typs ahd. hōhī zusammengefallen und bilden den Singular in der Regel einheitlich auf hōhī (vgl. Braune/Reiffenstein, Ahd. Gr. 2004, §231). Formen auf -ìn sind dagegen in althochdeutscher Zeit selten (vgl. Braune/Reiffenstein, Ahd. Gr. 2004, §228 Anm. 1), ${ }^{27}$ erst recht in mittelhochdeutscher, wo sie wohl weitgehend exklusiv in westoberdeutschen Quellen begegnen. ${ }^{28}$ Entsprechend gilt die auffällige Häufigkeit solcher Abstrakta auf -in (wie eben rǒtin) in dem Teil der Millstätter Handschrift, der auch Vom Rechte enthält, seit langem als ein Indiz für eine mögliche alemannische Entstehung oder Beeinflussung des Texts. Man hielt den Verfasser verschiedentlich „für einen - vielleicht in Kärnten lebenden - Alemannen“ (Ganz 1989, 1054); Edward Schröder (1891, 289-290, 297) wertete rǒtin als eine „alemann. form“. Friedrich Vogt (1921, 459-460) hielt es immerhin für möglich, „daß diese in erster linie gewiß alemannischen -în-formen als eine altertümliche erscheinung auch auf österreichischem sprachgebiet vorkommen“. Doch unabhängig davon, ob man rǒtin in Vom Rechte nun als einen morphologischen Archaismus oder als alemannische Form in südbairischer Schreibung wertet, bezeugt der Text jedenfalls die Existenz des Lexems für das Oberdeutsche in der Mitte des 12. Jahrhunderts.

Deutlich weiter zurück, noch vor die hochmittelalterliche Rodungsperiode, reicht freilich die Namenüberlieferung. Bereits ein exemplarischer Blick in zwei Quellen für den alemannischen Sprachraum zeigt, dass sich unter den massenhaft belegten Rodungsnamen des Früh- und Hochmittelalters ${ }^{29}$ zahlreiche Kandidaten für das Abstraktum ahd. riutī(n) finden, dessen Bedeutung offenbar früh genug konkretisiert wurde, ${ }^{30}$ um wie das Neutrum toponymisch Verwendung finden zu können. So erwähnen die kaiserlichen Besitzbestätigungen für das Kloster Einsiedeln in ottonisch-salischer Zeit je drei Orte mit dem Namen Riutin (bzw. Ríutin, Rîutin, Rutin). ${ }^{31}$

27 Im Altbair. endet der Sg. nach Schatz (1907, §114) stets auf -ī.

28 Vgl. die Nachweise bei Klein/Solms/Wegera (2009, § S66.2) für Adjektivabstrakta wie vinsterin, wüestin etc. So auch Paul, Mhd. Gr. (2007, §M18 Anm. 4). Einige mittelhochdeutsch-bairische Belege führt jedoch Weinhold (1883, §462) an.

29 Man vgl. allein das bei Förstemann (1916, Bd. 2) auf den Sp. 616-633 zusammengetragene Material.

30 Zur anzunehmenden Bedeutungsentwicklung vgl. Szadrowsky (1933, 64).

31 MGH DD O II. 24 (a. 972, Orig.), H II. 378 (a. 1018, Orig.), Ko II. 109 (1027, Kop. 15. Jh.) und H III. 36 (1040, Orig.). Die Namenschreibung der Diplomata ist bei der ältesten Urkunde wenig überzeugend („Rúttin“, „Ruttin“); vorzuziehen ist die Lesung des ZUB 1, Nr. 214; vgl. auf monasterium.net das Digitalisat des Diploms (Klosterarchiv Einsiedeln Urk. 17). Zur schwierigen Identifikation der Orte (bei Zürich, Tuggen und Ravensburg) vgl. etwa TUB 2, Nr. 1. 
Es handelt sich dabei ebenso um das Femininum wie bei einer Reihe von Ortsnennungen im frühmittelalterlichen Rotulus des Kanonikerstifts am Zürcher Großmünster. Dieser verzeichnet für den Raum Zürich eine Riutin bzw. Samilinis Riutin (wohl Rütihof b. Fallenden Brunnen auf dem Milchbuck), eine Tugilin Riutin (wohl der Hof im Düggel, südl. v. Zollikon) und eine Utin Riutin (nicht identifiziert): ${ }^{32}$

876-880 Kop. um 930 pater suus in Riutin (ZUB 1, Nr. 140); um 930 (?) Kop. um 950 in loco, qui dicitur Tugilin Riutin; iugera II cum aedificiis Samilinis Riutin (ZUB 1, Nr. 193); um 930/47 (zu 2. Hälfte 9. Jh.) ad Riutin et illa marcha (ZUB 1, Nr. 37); 946 de Samilinis Riutin (ZUB 1, Nr. 197); 947-954 De / in Samilinis Riutin; talem proprietatem, qualem Samilin in Riutin marcha... visus fuisset possidere; ad ipsum locum Riutin (ZUB 1, Nr. 199). - Für Samilinis Riutin findet sich sogar eine kurze Beschreibung des Rodungsvorgangs. Ein gewisser Sigihart, Vater des später namengebenden Samilin, charakterisiert sein mühsam erlangtes Eigengut in einer Urkunde von ca. 880 mit den Worten: proprietatem meam, quam labore proprio de incultis silvis extirpavi (ZUB 1, Nr. 141).

Die zitierten Namen lauten im Nominativ, Genitiv (Riutin marcha!) und im Dativus localis stets riutin und lassen damit das charakteristisch eintönige Singular-Paradigma des i-Abstraktums gut erkennen. Die weitere Entwicklung im Westoberdeutschen zeichnet sich dann bereits bei Notker deutlich ab: Im Singular setzt sich zunehmend der vokalische Auslaut durch; im Plural werden (wohl ausgehend vom Genitiv) erweiterte Formen verallgemeinert ${ }^{33}$ und eine klare Numerusprofilierung durchgeführt. So heißt es etwa zu Anfang des 14. Jhs. im Habsburger Urbar: ein ruti (dú giltet...) vs. die rutinen (Maag 1894, 306-307). Entsprechend lautet der Plural in rezentem Alemannisch Rü̈ti - Rü̈tine ${ }^{n}$ und ursprünglich auch im Schwäbischen Reute - Reutene ${ }^{n}{ }^{34}$ Ein von Lexer (s. v.) angesetztes schwaches Femininum mhd. riutine ist dagegen ein reines Phantomlemma. Die dafür gebotenen Belege (in den rüttinen etc.) sind allesamt reguläre Plurale zu riüte < riutī. Es gibt folglich keinen Grund, mit Ring $(2008,47)$ eigens eine ursprüngliche -inō-Bildung anzunehmen..$^{35}$

Festzuhalten bleibt, dass sowohl das Neutrum riuti als auch das Femininum riutī nach Ausweis der Namen bereits früh im Oberdeutschen in Gebrauch waren. Seit spätalthochdeutscher (-ríute bei Notker) bzw. frühmittelhochdeutscher Zeit (rǒtin in Vom Rechte) lassen sich beide auch appellativisch fassen. Welchem der beiden Lexeme die Belege im Mondseer Bibelglossar zuzuordnen sind, ist jedoch nicht sicher zu beantworten. Die allgemeine Festlegung auf riuti erscheint jedenfalls einigermaßen willkürlich.

32 Zur (nur „mit Vorbehalten“ möglichen) Identifikation der Orte vgl. Steiner (1998, 160); die Datierung der Urkunden entsprechend den Vorschlägen bei Steiner (1998, 52-66 u. 99-103).

33 Vgl. Braune/Reiffenstein, Ahd. Gr. (2004, §228 Anm. 3) und zum Alemannischen materialreich Weinhold (1863, §406-407); Szadrowsky (1933, §4).

34 Vgl. Schweiz. Id. (6, 1811); Schwäb. WB (5, 320),(inzwischen wohl nicht mehr gebräuchlich).

35 Auch das Schweiz. Id. $(6,1811)$ kennt für ein solches Lemma keine alten Belege und befindet: „Sicher meist nur der als Sg. gefasste Pl. von Rüti“. 


\section{Quellen}

Hamano, Akihiro (2016): Die frühmittelhochdeutsche Genesis. Synoptische Ausgabe nach der Wiener, Millstätter und Vorauer Handschrift. Berlin/Boston.

Hauthaler, Willibald/Martin, Franz (Hg.) (1910): Salzburger Urkundenbuch. Bd. 1: Traditionscodices. Salzburg.

Heeg-Engelhart, Ingrid (1990): Das älteste bayerische Herzogsurbar. Analyse und Edition. München. Höppl, Reinhard (1984): Die Traditionen des Klosters Wessobrunn. München.

Klose, Josef (2003): Die Urbare Abt Hermanns von Niederalteich. 2 Bde. München.

Lošek, Franz (1990): Notitia Arnonis und Breves Notitiae. Die Salzburger Güterverzeichnisse aus der Zeit um 800: sprachlich-historische Einleitung, Text und Übersetzung. In: Mitteilungen der Gesellschaft für Salzburger Landeskunde 130, 5-192.

Maag, Rudolf (1894): Das Habsburgische Urbar. Basel.

MGH DD = Monumenta Germaniae Historica. Diplomata (O[tto] II., H[einrich] II., Ko[nrad] II., H[einrich] III.), http://www.dmgh.de (Stand: 7.2.2017)

Schneider, Karin (1987): Gotische Schriften in deutscher Sprache. Vom späten 12. Jahrhundert bis um 1300. Textband. Wiesbaden.

Trad. Freising = Bitterauf, Theodor (1905-1909): Die Traditionen des Hochstifts Freising. 2 Bde. München.

Trad. Wessobrunn s. Höppl (1984).

TUB = Thurgauer Urkundenbuch (1924-1967). Hg. auf Beschluss und Veranstaltung des

Thurgauischen Historischen Vereins. 8 Bde. Frauenfeld.

ZUB $=$ Urkundenbuch der Stadt und Landschaft Zürich (1888-1957). 13 Bde. Zürich.

\section{Literatur}

Braune, Wilhelm/Reiffenstein, Ingo (2004): Althochdeutsche Grammatik I: Laut- und Formenlehre. Tübingen.

ANBÖ = Hausner, Isolde/Schuster, Elisabeth (1999-2014): Altdeutsches Namenbuch. Die Überlieferung der Ortsnamen in Österreich und Südtirol von den Anfängen bis 1200. 2 Bde. Wien.

AWB = Frings, Theodor/Große, Rudolf/Karg-Gasterstädt, Elisabeth/Lerchner, Gotthard/Schmid, Hans Ulrich (Hg.) (1968-): Althochdeutsches Wörterbuch. Auf Grund der von Elias von Steinmeyer hinterlassenen Sammlungen im Auftrag der Sächsischen Akademie der Wissenschaften zu Leipzig. Begründet von Elisabeth Karg-Gasterstädt und Theodor Frings. Berlin.

Baumann, Cornelia (1989): Altlandkreis Erding. München.

$\mathrm{BMZ}=$ Benecke, Georg Friedrich/Müller, Wilhelm/Zarncke, Friedrich. (1854-1866): Mittelhochdeutsches Wörterbuch. 3 Bde. Leipzig.

BStK = Bergmann, Rolf/Stricker, Stefanie (Bearb.) (2005): Katalog der althochdeutschen und altsächsischen Glossenhandschriften. Berlin/New York.

Davids, Hendrik (2000): Studien zu den substantivischen Bibelglossen des CIm 19440 aus Tegernsee. Ein Beitrag zur Erforschung der Bibelglossatur M. Göttingen.

DRW = Heidelberger Akademie der Wissenschaften (Hg.) (1914-): Deutsches Rechtswörterbuch. Wörterbuch der älteren deutschen Rechtssprache. Bis Bd. 3 hg. von der Preußischen Akademie der Wissenschaften. Bd. 4 hg. von der Deutschen Akademie der Wissenschaften. Bisher 12 Bde. Weimar. 
${ }^{1}$ DWB $=$ Deutsches Wörterbuch von Jacob Grimm und Wilhelm Grimm (1854-1960). 16 Bde. 1. Aufl. Leipzig. Nachdruck München 1984.

Förstemann, Ernst (1913-1916): Altdeutsches Namenbuch. Bd. 2: Ortsnamen. Bonn.

Ganz, Peter (1989): Vom Rechte. In: Ruh, Kurt (Hg.): Die deutsche Literatur des Mittelalters. Verfasserlexikon. Bd. 7. Berlin/New York, 1054-1056.

Gröger, Otto (1911): Die althochdeutsche und altsächsische Kompositionsfuge. Mit Verzeichnis der althochdeutschen und altsächsischen Composita. Zürich.

Hartl, Eduard (1930): Ein neues althochdeutsches Glossenfragment. In: Hartmann, Albert (Hg.): Festschrift für Georg Leidinger zum 60. Geburtstag. München, 95-101.

Klein, Thomas/Solm, Hans-Joachim/Wegera, Klaus-Peter (2009): Mittelhochdeutsche Grammatik. Teil III: Wortbildung. Tübingen.

Krahe, Hans/Meid, Wolfgang (1969): Germanische Sprachwissenschaft. Bd. III: Wortbildungslehre. Berlin/New York.

Kranzmayer, Eberhard (1956): Historische Lautgeographie des gesamtbairischen Dialektraumes. Lexer $=$ Lexer, Matthias (1872-1878): Mittelhochdeutsches Handwörterbuch. 3 Bde. Leipzig.

Maurer, Friedrich (1965): Die religiösen Dichtungen des 11. und 12 Jahrhunderts. Bd. II. Tübingen.

Meineke, Birgit (2009): Das Mondseer Bibelglossar. In: Bergmann, Rolf/Stricker, Stefanie (Hg.): Die althochdeutsche und altsächsische Glossographie. Ein Handbuch. Bd. 1. Berlin/New York, 619-634.

Meineke, Birgit (2013): Mondseer Bibelglossen. In: Bergmann, Rolf (Hg.): Althochdeutsche und altsächsische Literatur. Berlin/Boston, 263-271.

Paul, Hermann (2007): Mittelhochdeutsche Grammatik. Neu bearb. von Thomas Klein, HansJoachim Solms und Klaus-Peter Wegera. Mit einer Syntax von Ingeborg Schöbler, neubearb. und erw. von Heinz-Peter Prell. Tübingen.

Pimenova, Natalia (2002): Die semantische Stellung von deverbalen î(n)-Nomina im althochdeutschen Wortbildungssystem. In: Habermann, Mechthild/Müller, Peter 0./Munske, Horst Haider (Hg.): Historische Wortbildung des Deutschen. Tübingen, 137-158.

Prinz, Michael (2010): Vergessene Wörter. Frühe volkssprachliche Lexik in lateinischen Urkunden und Amtsbüchern. In: Jahrbuch für germanistische Sprachgeschichte 1, 292-322.

Prinz, Michael (i. Dr.): Vom „Roden der Seele“. Germ. *reupa- und Verwandtes.

Puchner, Karl (1951): Landkreis Ebersberg. München.

Reiffenstein, Ingo (2003): Aspekte einer Sprachgeschichte des Bayerisch-Österreichischen bis zum Beginn der frühen Neuzeit. In: Besch, Werner et al. (Hg.): Sprachgeschichte. Ein Handbuch zur Geschichte der deutschen Sprache und ihrer Erforschung. (Handbücher zur Sprach- und Kommunikationswissenschaft 2.3). Berlin/New York, 2889-2942.

Riecke, Jörg (1996): Die schwachen jan-Verben des Althochdeutschen. Ein Gliederungsversuch. Göttingen.

Ring, Uli (2008): Substantivderivation in der Urkundensprache des 13. Jahrhunderts. Eine historisch-synchrone Untersuchung anhand der ältesten deutschsprachigen Originalurkunden. Berlin/New York.

Schatz, J[osef] (1907): Altbairische Grammatik. Laut- und Flexionslehre. Göttingen.

Schmeller = Schmeller, Johann Andreas (1872-1877): Bayerisches Wörterbuch. 2., mit des Verfassers Nachträgen vermehrte Ausgabe. Hg. v. G. Karl Frommann. Bd. 1: München 1872. Bd. 2: München 1877 (wiederholt Nachdrucke: Leipzig 1939; Aalen 1961, 1966, 1973; München/ Wien/Aalen 1983; München 1985, 1996 usw.).

Schröder, Edward (1891) Rezension von: Carl Kraus: 'Vom rechte' und 'Die hochzeit'. Wien 1891. In: Anzeiger für deutsches Alterthum und deutsche Litteratur 17, 287-301.

Schröder, Werner (1957): Kritisches zu neuen Verfasserschaften Walahfried Strabos und zur 'Althochdeutschen Schriftsprache'. In: ZfdA 87, 163-213. 
Schützeichel, Rudolf (Hg.) (2004): Althochdeutscher und Altsächsischer Glossenwortschatz. Bearbeitet unter Mitwirkung von zahlreichen Wissenschaftlern des In- und Auslands. 12 Bde. Tübingen.

Schützeichel, Rudolf (2012): Althochdeutsches Wörterbuch. Berlin/Boston.

Schwäb. WB = Schwäbisches Wörterbuch (1904-1936). Bearb. v. Hermann Fischer. 6 Bde. Tübingen.

Schweiz. Id. = Schweizerisches Idiotikon. Wörterbuch der schweizerdeutschen Sprache (1881-) Begonnen von Friedrich Staub/Ludwig Tobler. Bd. I-XVII/Lfg. 224. Frauenfeld.

Seebold, Elmar (2008): Chronologisches Wörterbuch des deutschen Wortschatzes. Bd. II: Der Wortschatz des 9. Jahrhunderts. Berlin/New York.

Sehrt, Edward H. (1962): Notker-Glossar. Ein althochdeutsch-lateinisch-neuhochdeutsches Wörterbuch zu Notkers des Deutschen Schriften. Tübingen.

Speicher, Stephan (1986): „Vom Rechte“. Ein Kommentar im Rahmen der zeitgenössischen Literaturtradition. Göppingen.

Steiner, Hannes (1998): Alte Rotuli neu aufgerollt. Quellenkritische und landesgeschichtliche Untersuchungen zum spätkarolingischen und ottonischen Zürich. Freiburg/München.

StSGI. = Steinmeyer, Elias/Sievers, Eduard (Hg.) (1879-1922): Die althochdeutschen Glossen. 5 Bde. Berlin. Nachdruck Dublin/Zürich 1968-1969.

Starck, Taylor/Wells J. C. (Hg.) (1972-1990): Althochdeutsches Glossenwörterbuch (mit Stellennachweis zu sämtlichen gedruckten althochdeutschen und verwandten Glossen). Heidelberg.

Sullivan, Robert G (2001): Justice and the Social Context of Early Middle High German Literature. New York.

Szadrowsky, Manfred (1933): Abstrakta des Schweizerdeutschen in ihrer Sinnentfaltung. Frauenfeld.

Tiefenbach, Heinrich (1980): Bezeichnungen für Fluren im Althochdeutschen, Altsächsischen und Altniederfränkischen. In: Beck, Heinrich/Denecke, Dietrich/Jankuhn, Herbert (Hg.): Untersuchungen zur eisenzeitlichen und frühmittelalterlichen Flur in Mitteleuropa und ihrer Nutzung. Bd. 2. Göttingen, 287-322.

TNB = Thurgauer Namenbuch (2003-2007). Die Siedlungsnamen des Kantons Thurgau. Frauenfeld.

Vogt, F[riedrich] (1921): Zum Kürenberger. In: PBB 45, 459-467.

Weinhold, Karl (1863): Alemannische Grammatik. Berlin.

Weinhold, Karl (1883): Mittelhochdeutsche Grammatik. Paderborn.

Wiesinger, Peter (1970): Phonetisch-phonologische Untersuchungen zur Vokalentwicklung in den deutschen Dialekten. 2 Bde. Berlin.

Wiesinger, Peter (1992): Zur Morphologie der bairischen Ortsnamen im Althochdeutschen. In: Schützeichel, Rudolf (Hg.): Philologie der ältesten Ortsnamenüberlieferung. Heidelberg, 355400.

WMU = Kirschstein, Bettina/Schulze, Ursula (Hg.) (1994-2010): Wörterbuch der mittelhochdeutschen Urkundensprache auf der Grundlage des Corpus der altdeutschen Originalurkunden bis zum Jahr 1300. 3 Bde. Berlin.

Wunderle, Elisabeth (2010): Cgm 5248. Die sogenannten althochdeutschen Fragmente der Bayerischen Staatsbibliothek München. In: ZfdA 139, 197-221. 
